\title{
Extremely reduced supergroup F Wolbachia: transition to obligate insect symbionts
}

\author{
Sazzad Mahmood ${ }^{1}$, Eva Nováková1,2, Jana Martinů ${ }^{1,2}$, Oldřich Sychra ${ }^{3}$, Václav Hypša ${ }^{1}$
}

\author{
${ }^{1}$ Department of Parasitology, Faculty of Science, University of South Bohemia, České Budějovice, Czech \\ Republic \\ ${ }^{2}$ Institute of Parasitology, Biology Centre, ASCR, v.v.i., České Budějovice, Czech Republic \\ ${ }^{3}$ Department of Biology and Wildlife Diseases, Faculty of Veterinary Hygiene and Ecology, University of \\ Veterinary Sciences, Brno, Czech Republic
}

Author for correspondence: Václav Hypša, Department of Parasitology, University of South Bohemia, České Budějovice, Czech Republic, +42 387776 276, vacatko@prf.jcu.cz

\begin{abstract}
Wolbachia are widely distributed symbionts among invertebrates that manifest by a broad spectrum of lifestyles from parasitism to mutualism. Wolbachia Supergroup F is considered a particularly interesting group which gave rise to symbionts of both arthropods and nematodes, and some of its members are obligate mutualists. Further investigations on evolutionary transitions in symbiosis have been hampered by a lack of genomic data for Supergroup F members. In this study, we present genomic data for five new supergroup F Wolbachia strains associated with four chewing lice species. These new strains in different evolutionary stages show genomic characteristics well-illustrating the evolutionary trajectory which symbiotic bacteria experience during their transition to mutualism. Three of the strains have not yet progressed with the transition, the other two show typical signs of ongoing gene deactivation and removal (genome size, coding density, low number of pseudogenes). Particularly, wMeur1, a symbiont fixed in all Menacanthus eurysternus populations across four continents, possesses a highly reduced genome of 733,850 bp with a horizontally acquired capacity for pantothenate synthesis. Comparing with other strains showed wMeur1 genome as the smallest currently known among all Wolbachia and the first example of Wolbachia which has completed genomic streamlining known from the gammaproteobacterial obligate symbionts.
\end{abstract}

\section{Introduction}

Wolbachia present a unique example of diversity and phenotypic flexibility found within a single monophyletic group of bacterial symbionts. Originally described as a causative agent of cytoplasmic incompatibility in Culex pipiens mosquitoes, the genus is today known to be widely distributed among many arthropods and some nematode species (1). The diversity of their lifestyles spans from parasites to 
obligate mutualists. Phylogenetically, Wolbachia form several distinct clusters, usually called supergroups (2) which have developed their own characteristic features and tendencies. For example, while most supergroups seem to be specific to arthropods, few are reported exclusively from filarial nematodes. A particularly interesting group is supergroup $F$, the only supergroup known to infect both arthropods and nematodes (2-4). Moreover, some members of this supergroup are highly adapted strains with degraded genomes, which can maintain a mutualistic relationship with their hosts $(5,6)$.

The rapidly growing number of Wolbachia genome assemblies now allows for evolutionary and functional comparisons and identification of the characteristics underlying different life strategies $(4,7)$. However, the distribution of the available data across the supergroups and host taxa is extremely uneven and biased. The recent meta-analysis performed by Scholz et al. (1) included an impressive number of 1,166 Wolbachia genomes or genome drafts, but the majority of them $(1,018)$ originated from dipterans, almost exclusively from Drosophila (1,011). Similarly, regarding the taxonomic diversity of Wolbachia, 1,055 of the genomes represented supergroup $A$, while only 11 belonged to supergroup $F$ and originated from three different hosts. This uneven distribution of genomes is likely to reflect the difference in attention paid to model and non-model organisms, rather than the real diversity of Wolbachia. Occasional screenings suggest that the supergroups underrepresented by genomic data may encompass a high diversity of Wolbachia strains. As an example, supergroup F, currently represented by four genomes and genome drafts from two nematodes and two arthropods $(1,8-10)$, seems to contain a wide variety of Wolbachia from different hosts when screened for specific Wolbachia genes $(2,11-13)$.

Phthiraptera belong to the insect taxa which have been screened specifically for the presence of Wolbachia symbionts and seem to be frequently infected. Kyei-Poku et al. (14) performed a PCR-based screening of 19 species, encompassing both sucking lice of the suborder Anoplura as well as the chewing lice of the suborders Amblycera and Ischnocera. They showed that all the tested samples produced specific Wolbachia markers, in some cases suggesting the occurrence of multiple strains. Since the screening was based on specific phylogenetic markers, genomic data is not available for these symbionts. It is therefore difficult to hypothesize on the nature of these symbiotic relationships and role of these Wolbachia for the hosts. From Anoplura that feed exclusively on vertebrate blood, several obligate symbionts of different phylogenetic origins have been characterized, and for some, their role in provisioning B vitamins has been demonstrated (15-21). Of the sucking lice included in the Kyei-Poku et al. (14) screening, this is the case for Riesia in Pediculus and Phthirus (16, 22), and Legionella polyplacis in Polyplax serrata (20, 21). Since the mutualistic role of these symbionts is well established, it is likely that Wolbachia do not play a nutritional role in these lice. They may rather be accompanying commensals or even parasites, as shown in many other insects. In chewing lice, the situation is less clear. These ectoparasitic insects are likely not a monophyletic group (23) and their feeding strategies are more diverse than in Anoplura. While all chewing lice are ectoparasites living in the fur or feathers of their hosts, the source of food varies among the groups, and in several cases their diet may also include host's blood $(24,25)$. Currently, a single genome is available for a chewing louse symbiont (26). This symbiont, described from slender pigeon louse Columbicola wolffhuegeli, is phylogenetically related to the genus Sodalis within Gammaproteobacteria. Its genomic characterization revealed features resembling other obligate symbionts in insects, namely 
strong size reduction and shift of GC content $(797,418 \mathrm{bp}, 31.4 \%$ of GC), but did not provide any clear evidence for its function in the host (26).

In this study we investigate the nature of Wolbachia symbionts in several species of chewing lice. We focus primarily on the widely distributed species Menacanthus eurysternus. This chewing louse has cosmopolitan distribution (27) and is known to parasitize a broad spectrum of passeriform and several piciform bird species (24). This allows us to test the obligate nature of $M$. eurysternus-associated Wolbachia across a broad range of samples. To assess the genomic characteristics and capacity of the symbiont, we assemble metagenomic data from $M$. eurysternus and reconstruct the complete genome of its Wolbachia symbiont. Finally, for comparative reasons, we assemble additional genome drafts from several chewing lice samples with metagenomic data available in SRA database (28).

\section{Methods}

\section{Material and DNA extraction}

Samples of Menacanthus eurysternus were collected across a large geographic distribution (Supplementary data 1) from 2000 to 2016. For 16S rRNA gene amplicon analysis, DNA templates were extracted from 54 individuals using QIAamp DNA Micro Kit (Qiagen) (Supplementary data 1). DNA template for metagenomics was isolated from pool of 8 individuals collected from one specimen of Fringilla coelebs morelatti GA72. To avoid environmental DNA contamination, lice were washed with pure ethanol (3x for $30 \mathrm{~min}$ ) in Mini-rotator (Bio RS-24) and DNA was extracted with QIAamp DNA Micro Kit (Qiagen). Concentration of the isolate was quantified with Qubit 2.0 Fluorometer (Invitrogen, Carlsbad, CA, USA) and the integrity of DNA was verified on agarose gel electrophoresis (1,5\%). NEBNext ${ }^{\circledR}$ Microbiome DNA Enrichment Kit (New England BioLabs) was used for increasing the proportion of bacterial DNA (via the procedure of selective binding and disposing of methylated host DNA). Final DNA concentration was quantified with a Qubit 2.0 Fluorometer using High Sensitivity reagents.

\section{$16 S$ rRNA gene amplicon sequencing and analysis}

The diversity and distribution of microbial associates in Menacanthus eurysternus samples were assessed using a 16S rRNA gene amplicon sequencing protocol developed by our group (29). Briefly, multiplexing was based on a double barcoding strategy using fused primers with 12-bp Golay barcodes in forward primer 515F, and 5-bp barcodes within the reverse primer $926 \mathrm{R}(30,31)$. An $18 \mathrm{~S}$ rRNA gene blocking primer (29) was involved in all PCR reactions to ensure sufficient yields of 16S rRNA gene amplicons from the metagenomic templates.

M. eurysternus samples were part of a highly multiplexed library containing 384 samples altogether. In order to control for amplification bias and contamination, two positive controls (commercially purchased mock communities ATCC $^{\circledR}$ MSA-1000 ${ }^{\mathrm{TM}}$ and ATCC $^{\circledR}$ MSA-1001 ${ }^{\mathrm{TM}}$ ), and two negative controls for PCR amplification were processed along with $M$. eurysternus samples (complete metadata including barcodes are available in Supplementary data 1 . The purified library was sequenced on Illumina Miseq using V2 
chemistry with 500 cycles (Norwegian High Throughput Sequencing Centre, Department of Medical Genetics, Oslo University Hospital).

The raw fastq data were processed into the OTU table with an in-house workflow combining Usearch (32) and Qiime 1.9 (33) scripts as described previously (29). Taxonomic classification was assigned to individual OTUs using BLAST searches of representative sequences against the SILVA 138 database (as of February 2021). Non-bacterial OTUs and potential contaminants found in the negative controls were cleaned from the data via a series of decontamination processes using different levels of stringency to evaluate the overall pattern of Wolbachia dominance and ubiquity in $M$. eurysternus microbiomes. While the less stringent decontamination involved eliminating 12 OTUs shared by both negative controls, the strict decontamination removed every OTU found the negative controls (35 OTUs altogether). The details on the control profiles and eliminated OTUs can be found in Supplementary data 1. The decontaminated datasets were rarefied in 5 iterations at a level of 1000 and 2000 reads and imported into RStudio (34) using phyloseq package (35). Compositional heat maps were produced for the 20 most abundant OTUs and ordered to reflect the phylogenetic relationship among analyzed $M$. eurysternus samples (complete COI phylogeny available in Supplementary figure 1).

Metagenomic sequencing and assembly (Menacanthus eurysternus)

The shotgun genomic libraries were prepared from the enriched gDNA of $M$. eurysternus GA72 sample using the Hyper Library construction kit from Kapa Biosystems (Roche). The library was sequenced in a multiplexed mode on one SP lane of NovaSeq 6000 for 251 cycles from both ends of the fragments. Fastq files were generated and demultiplexed with the bcl2fastq v2.20 Conversion Software (Illumina). The quality of 145,910,396 paired reads was checked with FASTQC and the data were trimmed by the Bbduk tool (36) to a minimal phred score of 20. Spades with the option --meta was used to assemble the metagenome. Initially, Wolbachia contigs were identified by blastn searches (37) using the complete set of genes from the Cimex lectularius symbiont wCle (ACC) as a query. Wolbachia origin of the preselected contigs was verified by blastn searches against the NCBI nt database. The amplicon analyses indicated the presence of two different Wolbachia strains. Based on the considerable length difference between the first contig and the rest of Wolbachia contigs (732,857 bp vs 26,534 and less) and different GC contents $(28 \%$ and $>33 \%$ ), we hypothesized that the first contig may be almost complete genome of one strain, while the others represent a second strain. To test this possibility by closing the genome of the first Wolbachia strain, we used two approaches. First, we extended the longest contig by aTRAM 2.0 (38) and closed it into a $733,850 \mathrm{bp}$ long circular sequence. Second, using specific primers designed based on the longest contig, we sequenced the missing part and completed the genome into a circular sequence identical with the result from aTRAM based approach. The remaining 189 contigs were considered as parts of the second strain of Wolbachia.

Screening and assembly of chewing lice SRA

To check for presence of Wolbachia in other chewing lice, we screened the available metagenomic data in SRA (28) (Supplementary table 1). Assembling of the reads and detection of Wolbachia were done in the same way as for M. eurysternus (described above). To identify candidate Wolbachia contigs, we used two 
genomes as queries, $w C l e$ and the newly assembled complete genome from $M$. eurysternus. Of five assemblies in which Wolbachia contigs were identified, two contained only a few short contigs with low coverage and were not included in the subsequent analyses (Supplementary table 1). For the remaining three assemblies we extracted Wolbachia genome drafts with different degree of fragmentations (from 9 contigs in Meromenopon meropis to 386 in Alcedoecus sp.). Since their sizes did not deviate from the common size of the other Wolbachia genomes (Table 1) and the completeness assessed by BUSCO was also comparable to other Wolbachia (Supplementary table 2; see below for BUSCO analyzes), we considered these sets of contigs as representative genome drafts.

Completeness assessment and annotations were done for both $M$. eurysternus strains and the three SRAderived strains by the same procedure. Completeness was assessed in BUSCO v5.1.2 with two different references, rickettsiales_odb10.2019-04-24 and proteobacteria_odb10.2019-04-24. Functional annotations of the genes were obtained by RAST (39). The presence of phage-related sequences was further checked by PHASTER (40). Potential pseudogenes were identified by Pseudofinder (41) based on annotation obtained from Prokka (42). Possible horizontal gene transfers (HGT) were identified by diamond blastx (43) against NCBI nr database with complete set of annotated genes as queries, e-value set to 10 and number of hits to five. Assignment of the genes to clusters of orthologous groups (COGs) was done in web-based eggNOG-Mapper (44). To visualize sharing of the genes across the supergroup F strains, we plotted results of the orthofinder analysis by UpSetR package of $R(45)$.

\section{Phylogeny}

To determine the position of the new strains, we designed two different matrices. First, since our preliminary analyses suggested that the new strains belonged to supergroup $F$, the "fbpA_coxA" nucleotide matrix was built to represent this supergroup. The matrix contained $48 \mathrm{~F}$ strains, and 23 additional strains representing other supergroups. The genes were retrieved from NCBI (https://www.ncbi.nlm.nih.gov/), pubMLST (46) and our new assemblies (Supplementary table 3). Alignment was done in MUSCLE (47). The web-based IQ-TREE tool (48) was used to select the best models ( $\mathrm{TN}+\mathrm{F}+\mathrm{I}+\mathrm{G} 4$ and $\mathrm{TVM}+\mathrm{F}+\mathrm{G} 4$ models for $f b p A$ and $\operatorname{cox} A$ respectively) and to perform phylogenic analysis. To verify the position of the new strains within supergroup $F$, we designed an amino acid "multigene matrix", restricted to the strains for which genomic data are available. This set contained all available genomes for supergroup $\mathrm{F}$ and several additional genomes representing other major supergroups (Supplementary table 3). For the included genomes, we identified 101 shared single copy orthologs by Orthofinder (49), aligned them in MUSCLE (47) and removed the unreliably aligned sites by Gblocks (50). The final concatenated matrix containing 23,218 positions was analyzed by two different approaches. Maximum likelihood analysis was done in IQ-TREE with $\mathrm{HIVb}+\mathrm{F}+\mathrm{I}+\mathrm{G} 4$ selected as best model. Since our data contained several long-branch sequences we used in addition PhylobayesMPI (51) with CAT-GTR model to minimize possible artifacts (52). This analysis was run for 50,000 generations under two different coding systems, first coding for each amino acid, second with amino acids recoded by the Dayhoff6 system. 


\section{Genomic and metabolic comparisons}

Genomic analyses and comparisons were done for the nine strains of supergroup $F$ for which complete genomes or drafts are available (Supplementary table 3). Average nucleotide diversity (ANI) was calculated using a web-based ANI calculator (53). Synteny of the genomes was analyzed in Mauve (54) implemented in Geneious (55). Assessment of metabolic capacities was done using the web-based tools Blastkoala and KEEG mapper (56). To obtain a metabolic overview comparable with other Wolbachia supergroups, we adopted the scheme used by Lefoulon et al. (10) and extended its content with comparison of amino acids synthesis.

\section{Results}

Amplicon screening of M. eurysternus

On average, 16S rRNA gene sequencing yielded 6,686 reads per sample under less stringent decontamination and 5,911 reads under the strict decontamination (see Materials and methods). The mock communities yielded, on average, 20,876 reads for the equally composed samples and 48,356 for the staggered communities. We were able to recover the expected profiles for both equal and staggered DNA template, including overrepresentation of Staphylococcus epidermidis (ATCC 12228) and vast underrepresentation of Rhodobacter sphaeroides (ATCC 17029) reported previously by the manufacturer (Supplementary data 1). Within the staggered communities, we retrieved all three extremely low abundant taxa $(0.04 \%$, Supplementary data 1$)$. The presence of an eleventh OTU of the genus Granulicatella however pointed out marginal (tens of reads) well-to-well contamination between positive and negative controls (details in Supplementary data 1). In all the datasets decontaminated under different stringency (see Materials and methods and Supplementary figure 2), Wolbachia OTUs clearly dominate $M$.

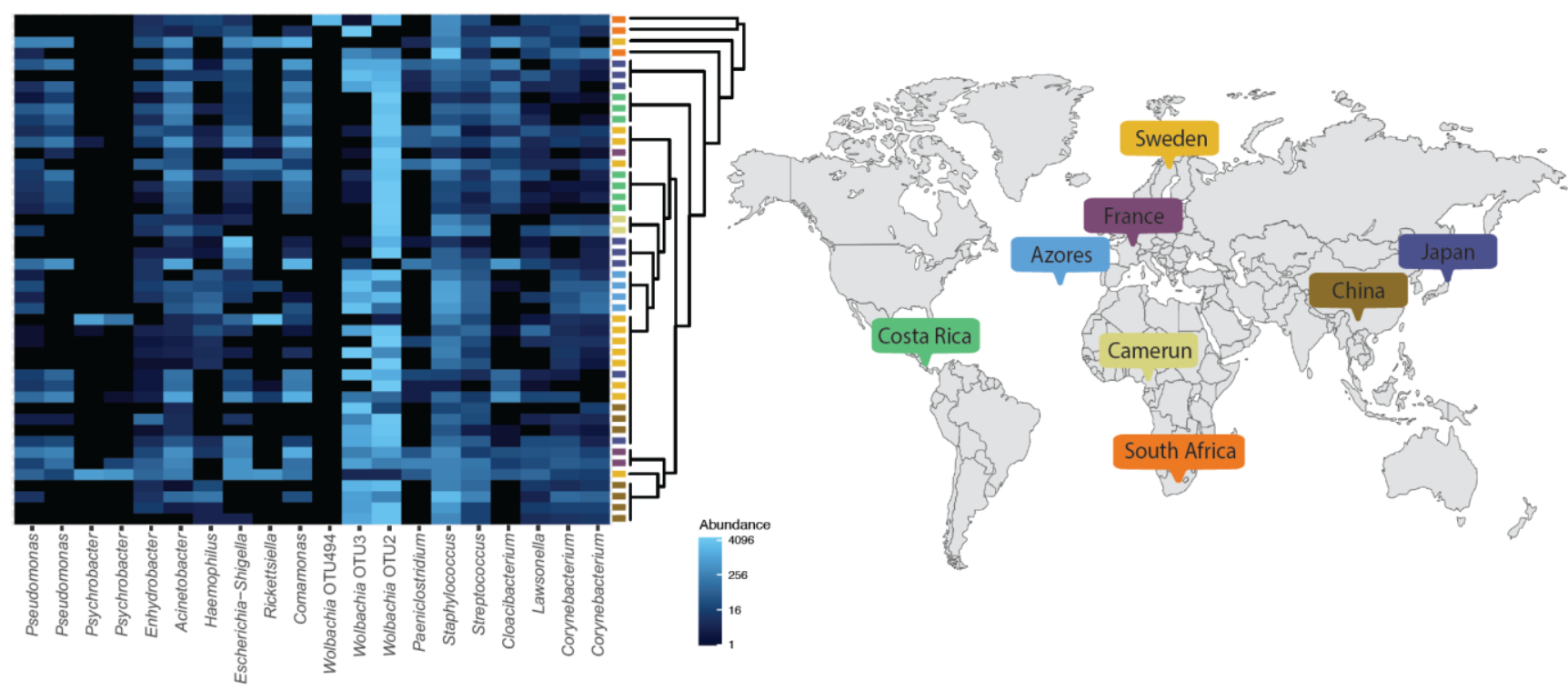

Figure 1: Compositional heat map produced for the 20 most abundant OTUs. The samples are ordered to reflect the phylogenetic relationships among analyzed $M$. eurysternus samples (complete COI phylogeny available in Supplementary Figure 1), and color coded according to their geographical origin. 
eurysternus microbiomes. While the analyzed individuals generally associate with a single Wolbachia (OTU2), some show a dual Wolbachia infection. However, the number of Wolbachia OTUs does not correlate with either geographic or phylogenetic origin of the analyzed hosts (Figure 1). Although few individuals in Figure 1 seemingly lack Wolbachia, these samples did not meet the rarefaction thresholds of 1000 and 2000 reads in the strictly decontaminated dataset (Supplementary figure 2) thus confirming a robust pattern of Wolbachia ubiquity across diversified populations of $M$. eurysternus.

\section{Metagenomic assemblies and genomes characterization}

Assembly of the M. eurysternus metagenomic data (sample GA72) contained two different Wolbachia strains. One strain (designated as wMeur1) was assembled into a single 732,857 bp long contig and closed into a complete circular genome using aTRAM extension to $733,850 \mathrm{bp}$. An identical sequence was

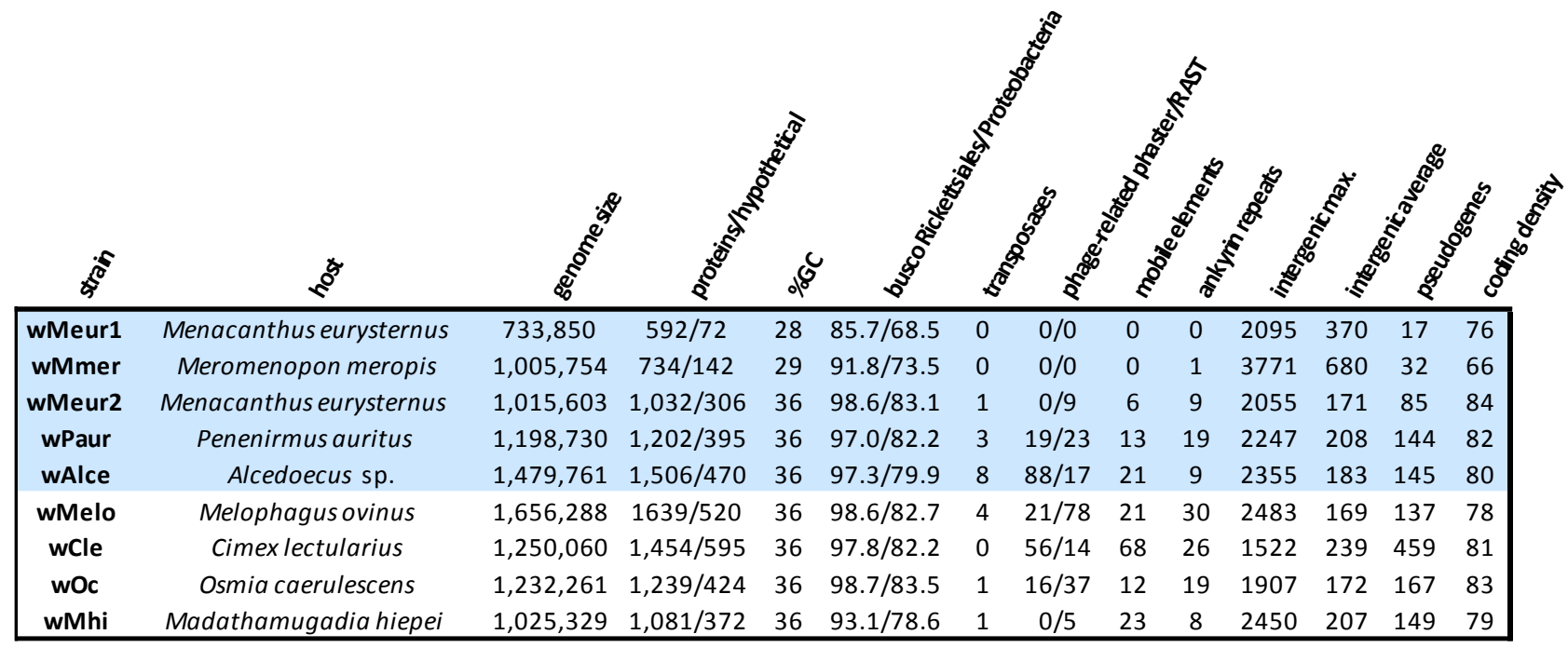

obtained using PCR with specific primers. The second strain, wMeur2, was fragmented into 189 contigs. Screening of the 36 chewing lice metagenomic data available in SRA database revealed additional three strains of Wolbachia, designated hereafter as wAlce, wPaur, and wMmer from Alcedoecus sp., Penenirmus auritus, and Meromenopon meropis, respectively. These genomes could only be assembled as drafts, composed of 9 (wMmer) to 386 (wAlce) contigs. The BUSCO assessments indicate that these fragmented genomes are complete or almost complete (SupplementaryTable2). When assessed against Rickettsiales database, the average completeness was 95\% (85.7\%-98.7\%). The assessment against Proteobacteria, performed to provide direct comparison with Lefoulon et al. (10), produced considerably lower average of $78.9 \%(68.5 \%-83.5 \%)$ corresponding to the results of Lefoulon et al. (10). For the wAlce strain, Busco predicted a higher degree of possible duplications, indicating that this assembly may not contain a single Wolbachia strain but could rather be a mixture of two closely related strains. wMeur1 and wMmer display unusual, derived features. Particularly, wMeur1 is only $733,850 \mathrm{bp}$ long with GC content of $28 \%$. Based on RAST annotation it contains 592 protein coding genes, 3 rRNA genes, and 35 tRNAs. wMmer genome is 
considerably longer (1,005,754 bp when concatenated) but with similarly low GC content (28.6\%). Both genomes form long branches in phylogenetic trees (Figure 2, supplementary figures 3,4 ) and possess characteristics typical for obligate symbionts. Beside the low GC content, they do lack transposase sequences, phage-related sequences, and mobile elements. Ankyrin repeats are not present in wMeur1 and only one instance was detected in wMmer (Table 1). The genomes of the remaining three strains resembled the previously reported genomes of supergroup $F$, containing transposase sequences, phagerelated sequences, mobile elements, and ankyrin repeats (Table 1 ).

Orthofinder placed most of the protein coding genes from the five new strains into orthogroups shared with the other included strains from supergroup F. Overlap comparison between the genomes showed a

A

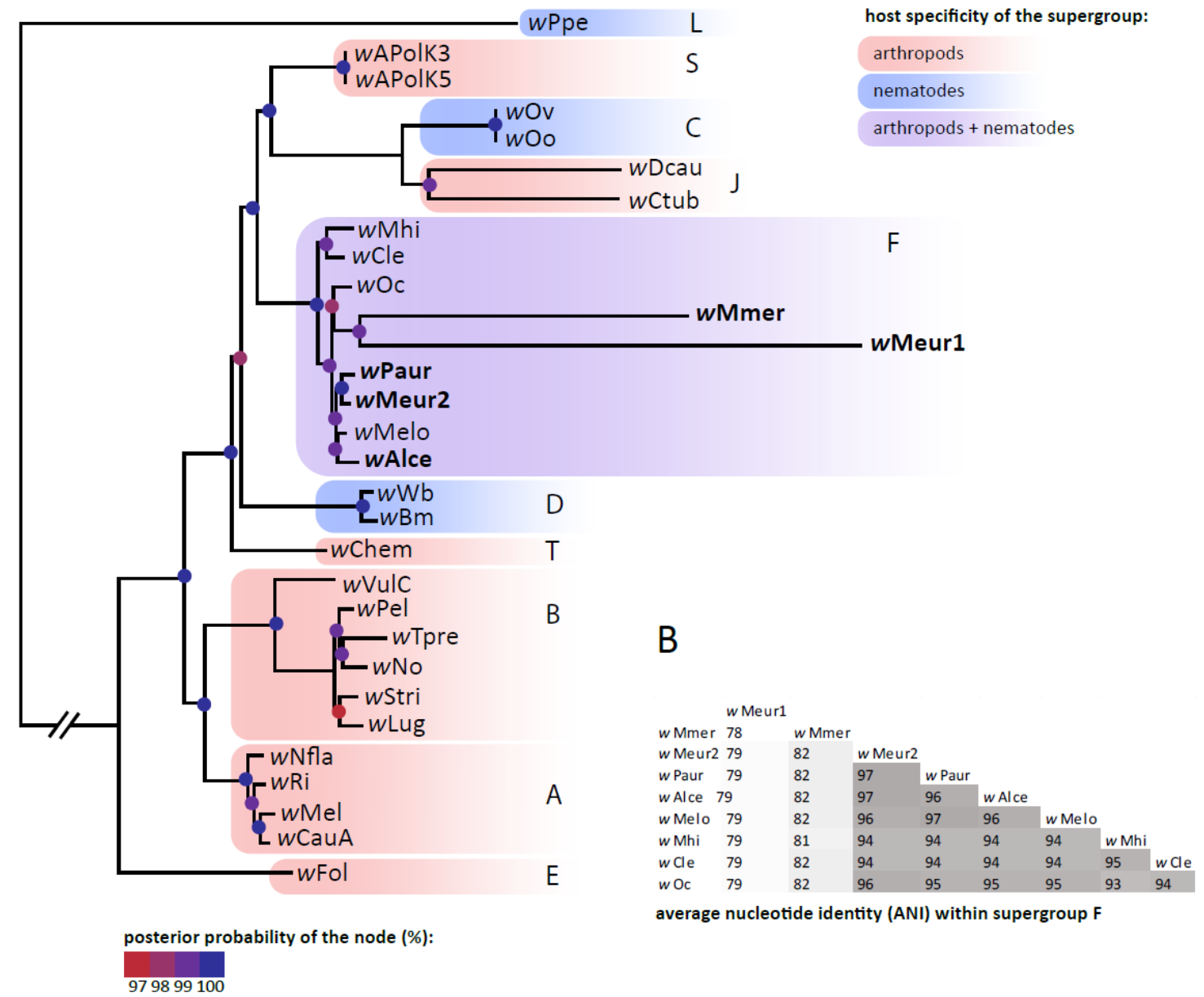

Figure 2: A - Phylogenetic relationships within the supergroup F derived for the available genomes ("multigene matrix" analyzed by Phylobayes). The new strains printed in bold. Posterior probabilities of the nodes are indicated by the colored dots. Super Supergroups are designated by the capital letters at the branches or clusters. B - Average nucleotide identity among the supergroup F genomes.

high proportion of genes shared by all or most strains (Figure 3; Supplementary data 2). It also revealed that wMeur1 and wMmer do not share any unique genes in exclusion of other genomes, despite their close phylogenetic relationship. On the other hand, while sharing a high proportion of genes, the strains 
displayed very limited degree of synteny (Supplementary figure 5). For example, Mauve analysis of closely related wMeur1 and wMmer produced 159 local collinear blocks, the longest spanning $18 \mathrm{~kb}$.

\section{Horizontal transfer of genes for pantothenate synthesis}

Orthofinder also identified a set of genes, each unique for a single strain, mostly annotated as hypothetical proteins (Supplementary table 4). A particularly interesting case is presented by three pantothenate synthesis related genes (panB, panG and panC) found exclusively in wMeur1. The only Wolbachia homologues found by diamond blastx were genes from wClefT (NZ_CP051156), a strain infecting the cat flea Ctenocephalides felis (8). The other closest relatives originated from phylogenetically distant bacteria, including several symbiotic forms. In the phylogenetic analysis of the closest homologues retrieved by blast from NCBI, all three wMeur1 pantothenate genes clustered as sister taxa to their wCfeT homologues (Supplementary figure 6).

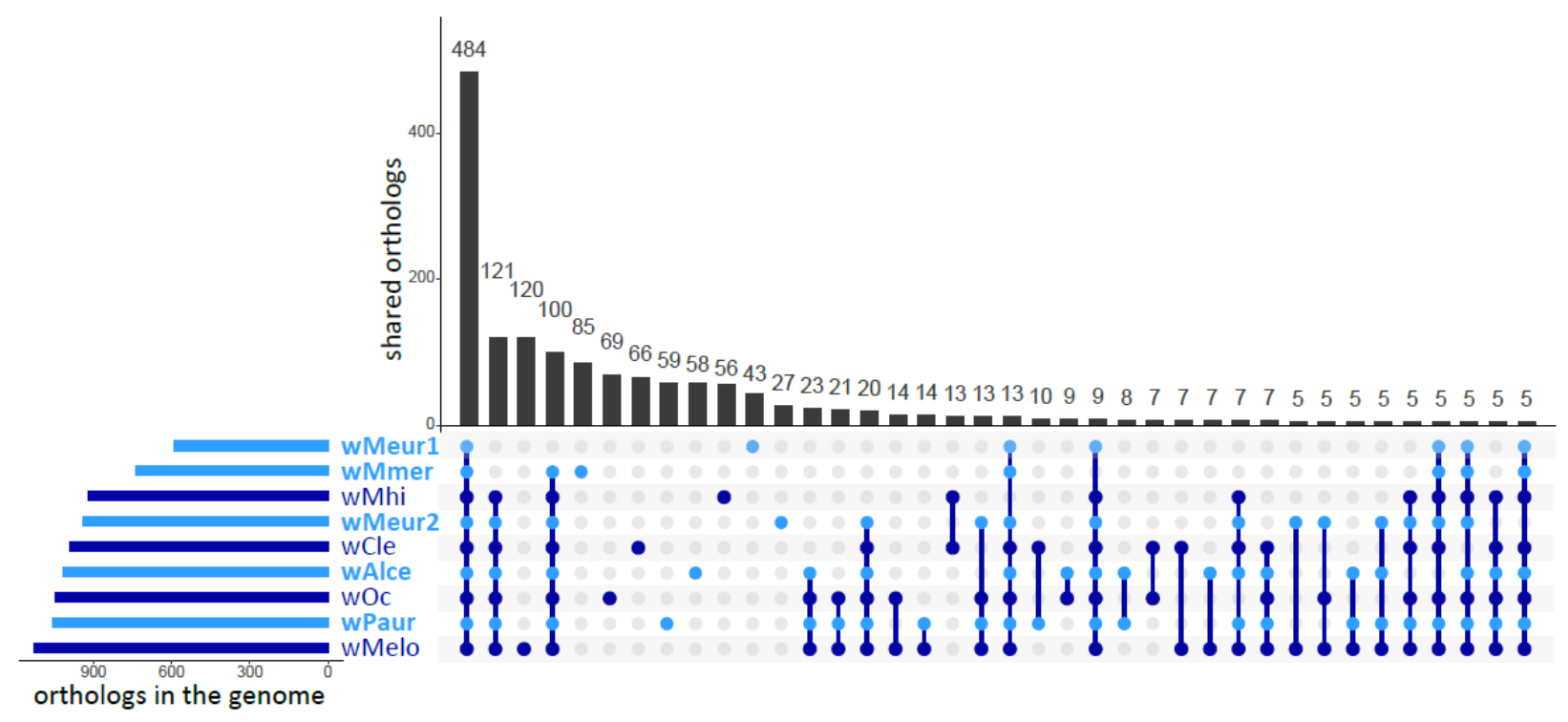

Figure 3: Orthogroups shared by the supergroup F Wolbachia genomes. Data for the new strains from chewing lice are printed in light blue.

In wMeur1, the three pantothenate genes were the only instances of apparent horizontal transfers. In the other four chewing lice strains of Wolbachia, the search for HGT did not reveal horizontally transferred genes with recognized metabolic function. Majority of the genes provided blast hits from other Wolbachia strains, while the few instances of the non-Wolbachia origin included mostly ankyrins, transposases, and hypothetical proteins (SupplementaryData3).

\section{Comparison of metabolic capacities}

Reconstruction of metabolic capacities shows high consistency across supergroup $F$ genomes (Supplementary table 5). The main differences are found in the wMeur1 genome and, to a lesser extent, in the $w \mathrm{Mmer}$ genome. Both genomes lost a high portion of recombination genes and $\mathrm{ABC}$ transports. The smaller wMeur1 also lost genes for two B vitamin pathways retained in other strains (pyridoxine and folate) but acquired three genes for pantothenate synthesis by horizontal transfer (see above). All genomes show very limited capacity for amino acid synthesis. They only retain a near-complete pathway 
for lysine (leading probably to synthesis of peptidoglycan rather than lysine) and the glyA enzyme allowing for interconversion of serine and glycine.

\section{Phylogenetic relationships of the Wolbachia symbionts}

All phylogenetic analyses of both matrices placed the newly described Wolbachia strains invariantly within the supergroup F (Figure 2, Supplementary figures 3,4 ). The two most derived strains wMeur 1 and $w M m e r$ formed extremely long branches within the supergroup, comparable only to those of the nematodeassociated mutualists from the supergroups J. In ML analysis of the "multigene" matrix these two long branches clustered at the base of the supergroup F (Supplementary figure 4), while in Phylobayes analysis they were nested among the other supergroup F taxa (Figure 2). The remaining three strains, wMeur2, wAlce, wPaur, were placed on considerably shorter branches, comparable to the rest of Wolbachia included in the analysis.

\section{Discussion}

\section{New highly derived members of supergroup $F$}

The new Wolbachia supergroup F strains described here from four species of chewing lice represent two remarkably different types of symbiont genomes. Three of them (wMeur2, wPaur, wAlce) resemble the other Wolbachia strains of supergroup $\mathrm{F}$ in their genomic characteristics (size above $1 \mathrm{Mb}$, average GC content between $35 \%$ and $37 \%$, presence of phage-related sequences, mobile elements, transposases, ankyrin repeats, etc.). This close similarity is also reflected in the short branches they form in the phylogenetic trees (Figure 2, Supplementary figures 3,4). However, two additional strains (wMeur1, wMmer) show very specific and derived traits, unique within the context of the whole Wolbachia diversity. Particularly, wMeur1 represents the first highly reduced, insect-associated Wolbachia strain with characteristics typical for obligate mutualists known from other bacterial groups $(20,57,58)$. It also provides another candidate example of transition towards mutualism by horizontal gene transfer $(20,59)$. This modifies the view that the arthropod associated strains of Wolbachia generally possess larger genomes, richer with transposable elements, prophage-related genes or repeat-motif proteins than their nematode-associated relatives $(4,60)$. With its 733,850 bp length wMeur1 is currently the smallest known genome among Wolbachia, almost $100 \mathrm{~kb}$ shorter than its nematode-associated "predecessors" wCtub and wDcau (10). The second derived strain, wMmer, possesses a genome larger than wMeur1, but shows similar signs of strong degeneration: both strains lack phage-related sequences, mobile elements, transposases and ankyrin repeats (with exception of one ankyrin repeat found in wMmer).

\section{Phylogenetic relationships}

The highly derived nature of wMeur1 and wMmer genomes is also apparent from the low ANI values, when compared to their close relatives, and the long branches they form in phylogenetic trees (Figure 2). In principle, such long branches may distort phylogenetic analyses. This phenomenon is particularly dangerous when analyzed taxa differ considerably in their nucleotide composition, e.g., when symbiotic genomes with extremely low GC content are included. In our study, the placement of these two symbionts 
within supergroup $\mathrm{F}$ is supported by several indications. First, this placement is not likely to be affected by long branches since no other members of supergroup $F$ possess long branches which would attract the wMeur1+wMmer pair. Second, the position of the two genomes as sister taxa has been retrieved with high support from all analyses, including the phylobayes analysis which is particularly resistant to this type of artifact (52).

Phylogenetic relationships within supergroup F suggest that at least the short-branched Wolbachia strains were acquired independently by their chewing lice hosts. For example, the two strains from philopterid lice, wAlce and wPaur, do not cluster as sister taxa in any of the analyses. While the tree resolution is rather poor and does not provide clear evidence, horizontal transfers within the $F$ supergroup have been deduced previously, e.g., between isopods and termites (13). This apparent lack of a coevolutionary signal is also concurrent with the absence of Wolbachia in all other screened SRA data for chewing lice (discussed below), and with the presence of a phylogenetically distant gammaproteobacterial symbiont in Columbicola wolffhuegeli $(26,61)$.

Distribution of the Wolbachia in chewing lice species

In our study, we found Wolbachia contigs in five out of the 36 assemblies of the SRA datasets. This is in sharp contrast with the results reported by Kyei-Poku et al. (14). In their screening, focused on Wolbachia in lice, these authors showed the presence of these bacteria in all 19 tested species (interestingly, none of them from supergroup F, all falling into A and B). However, their approach was based on PCR amplification of selected genes using specific primers. It is likely that this method can detect Wolbachia even if present in extremely low numbers. In contrast, the WGS-based approach will only produce data for the dominant bacteria in the microbiome. This is also reflected in the amplicon analyses, which revealed considerably more bacterial taxa in each individual $M$. eurysternus sample (Figure 1). This ambiguity raises the question, which of the Wolbachia previously detected in lice are parasites (known to be broadly distributed across arthropod taxa) and which may possibly represent the comparatively rarer instance of obligate mutualist.

Highly reduced wMeur1: transition to obligate symbiosis by HGT of pantothenate synthesis genes?

The genomic comparisons of the new strains are entirely consistent with their phylogenetic patterns. While the short-branched strains are closely similar in their genome characteristics, the two most derived genomes ( $w$ Meur1 and wMmer) differ in many aspects. They both underwent considerable deterioration of the recombination and transport systems and unlike the other strains, they lack the elements related to the genomic dynamics and fluidity (phages, transposases, mobile elements). Such evolutionary trends accompanying the reduction in genome size are well known from many obligate insect symbionts from gammaproteobacteria (62) but are much less common in Wolbachia. For example, several Wolbachia strains have been suggested to establish mutualistic relationships with their hosts after acquiring complete biotin operon $(8,59)$. Within insect hosts, these systems include $w C l e$ in the bedbug Cimex lectularius, wCfeT in the flea Ctenocephalides felis (8), or two Wolbachia strains from Nomada bees (63). In the latter, Wolbachia phylogeny even suggests co-divergence across several host species, typical for mutualistic obligate symbionts. However, in all these insects, Wolbachia symbionts retain genomes which exceed $1 \mathrm{Mb}$ and contain many mobile elements. As pointed out by Driscoll et al. (8), $w C l e$ and $w C f e T$ even possess 
extremely high numbers of pseudogenes (see table1 for $w C l e)$. The wMeur1 strain presented here differs from these insect symbionts by a dramatic reduction and "cleansing" of its genome. It is not only the smallest known genome among Wolbachia but also the first insect Wolbachia with genomic characteristics typical for obligate mutualists.

These features bring into question the role of the wMeur1 strain in its host. Unlike the above examples of presumably mutualistic Wolbachia, the wMeur1 strain does not possess genes required for biotin

A

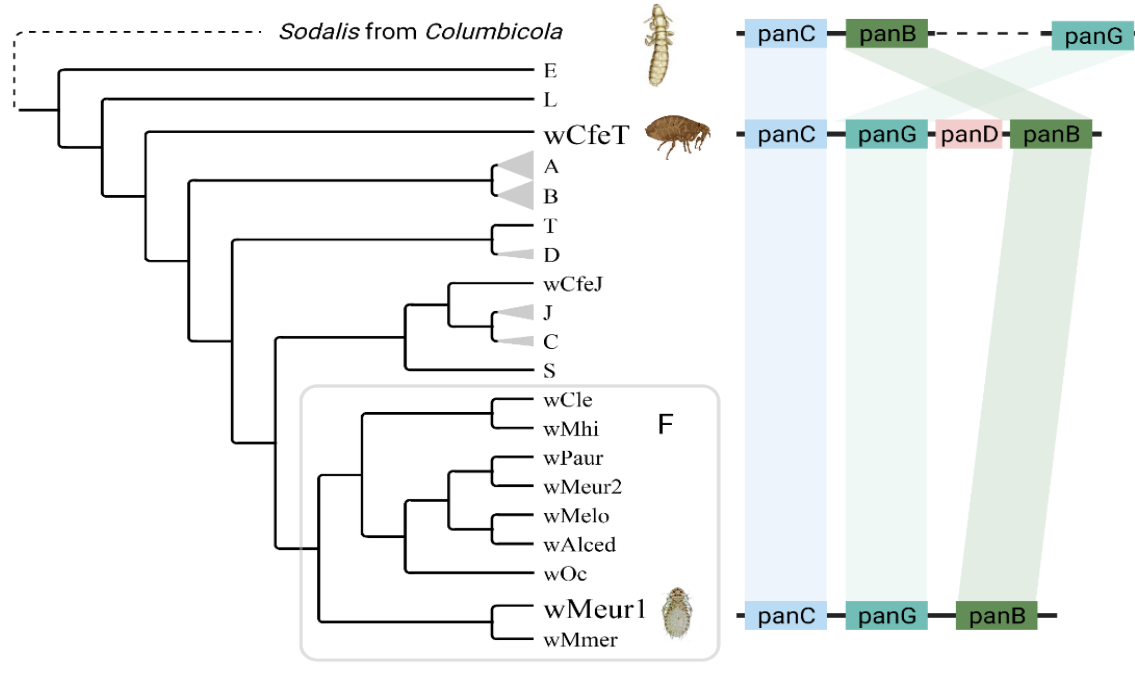

B

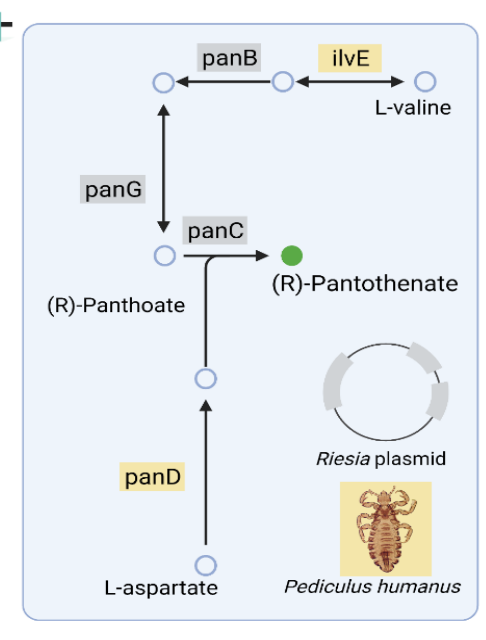

Figure 4: Distribution of the pantothenate synthesis related genes in the Wolbachia genomes. A - The genes mapped on a schematic phylogeny tree. B - overview of the pantothene synthesis pathway in Pediculus humanus and its symbiont Riesia pediculicola (reconstruction based on KEGG database).

synthesis. Of the other vitamin B pathways, often considered to be of potential importance in nutritional symbionts, it only retains the production of riboflavin, a pathway conserved across many Wolbachia strains (64). However, a striking metabolic difference between wMeur1 and all other supergroup $F$ strains is the presence of three genes required for synthesis of pantothenate, most likely acquired by horizontal gene transfer (HGT). The blast-based HGT analysis retrieved the most closely similar homologue from wCfeT, a Wolbachia strain from cat flea Ctenocephalides felis, while the other retrieved homologues belonged to other, often phylogenetically distant bacterial groups (Supplementary data 3). In the phylogenetic analysis, all three genes from wMeur1 and wCfeT formed closely related sister taxa (Supplementary figure 6). Remarkably, the same triad of genes is also present in the genome of a Sodalis-related symbiont from another chewing louse, C. wolffhuegeli (Figure 4; NCBI bioproject PRJNA692390). When characterizing metabolic capacity of this bacterium, Alickovic et al. (26) mainly addressed the issue of keratin digestion and concluded that no clear metabolic role can be deduced from the symbiont's genome content. Similarly, in our new strains, we failed to detect production of enzymes with keratinase activity, and we observed almost complete loss of capacity for amino acid synthesis (Supplementary table 5). However, the presence of the three pantothenate related genes in $C$. wolffhuegeli symbiont and their HGT acquisition 
bioRxiv preprint doi: https://doi.org/10.1101/2021.10.15.464041; this version posted October 19, 2021. The copyright holder for this preprint (which was not certified by peer review) is the author/funder, who has granted bioRxiv a license to display the preprint in perpetuity. It is made available under aCC-BY-NC-ND 4.0 International license.

by wMeur1 suggests that production of this vitamin might be at least part of the metabolic function in these obligate mutualists.

While the triad panB, panG, and panC forms a core of pantothenate synthesis, full functionality of the pathway requires two additional genes, ilvE and panD (Figure 4). These genes are missing in the wMeur1 genome but are very likely present in the genome of the host. The phylogenetically closest system with

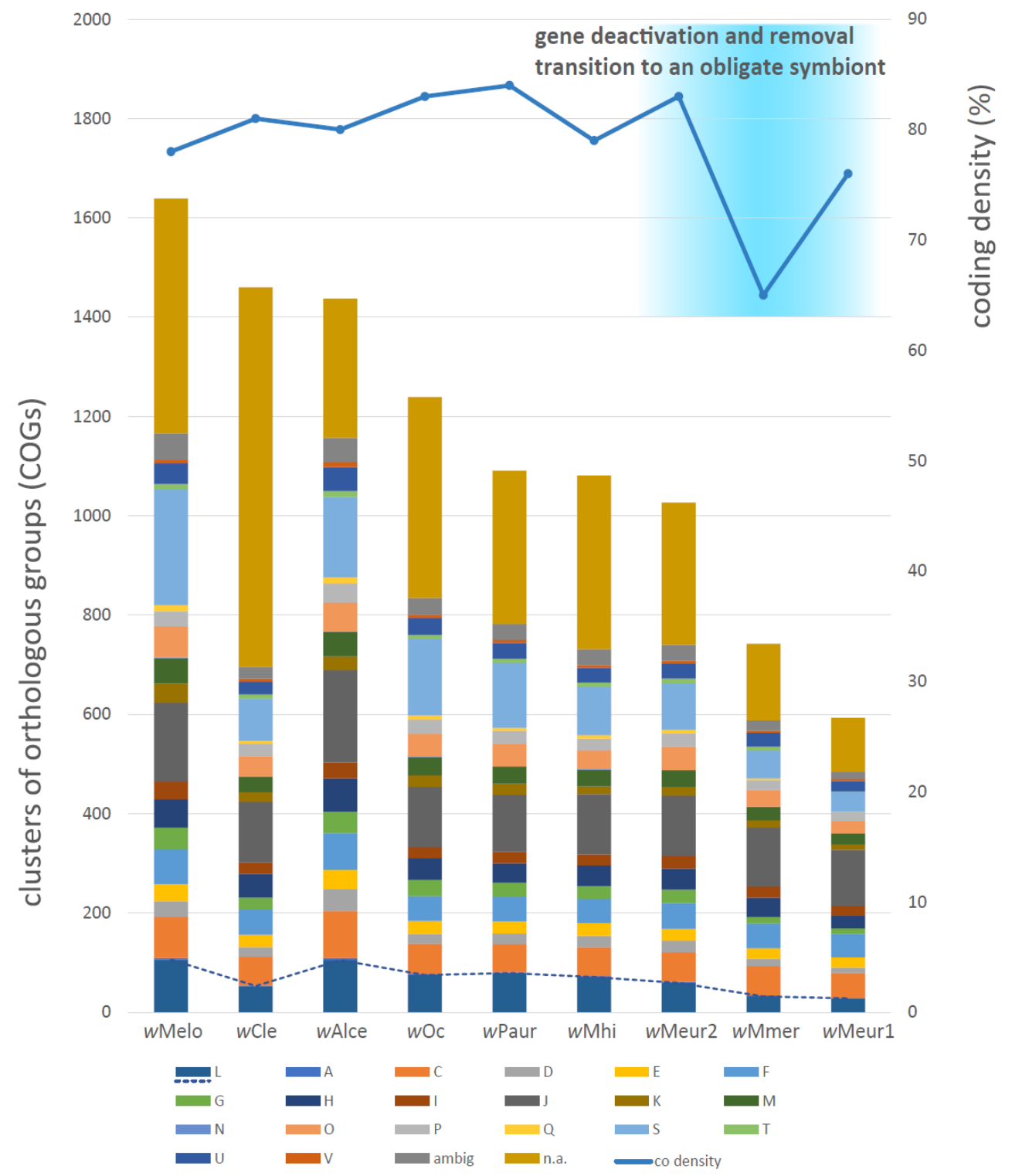

Figure 5: Comparison of the supergrup F Wolbachia genomes in respect to coding density and numbers of genes in different COGs. A - RNA processing and modification, C - Energy production and conversion, D - Cell cycle control and mitosis, E - Amino Acid metabolis and transport, F - Nucleotide metabolism and transport, G - Carbohydrate metabolism and transport, H - Coenzyme metabolism, I - Lipid metabolism, J Translation, K - Transcription, L - Replication, recombination and repair, M - Cell wall/membrane/envelop biogenesis, $\mathrm{N}$ - Cell motility, $\mathrm{O}$ Post-translational modification, protein turnover, chaperone functions, P - Inorganic ion transport and metabolism, Q - Secondary Structure, T - Signal Transduction, U - Intracellular trafficing and secretion, V - Defense mechanisms, S - Function Unknown, ambig - assigned to more than one category, n.a. - not assigned, co density - curve showing coding density. Dashed line highlights differences in the L category related to replication, recombination and repair. 
fully characterized genomic capacities is the symbiosis between human louse Pediculus humanus and its symbiont Riesia pediculicola (65). In this blood feeding insect, Riesia provides the same three genes (panB, panG and panC located on plasmid) while ilvE and panD are present in the host genome (in Figure 4 shown on simplified reconstruction based on the KEGG database). Our blast screening of the $M$. eurysternus assembly shows that these genes are also present in the wMeur1 host and suggests the complete pantothenate pathway is therefore functional.

According to the hypothetical scenario introduced by Lo et al. (66), symbiogenesis involves two bouts of dramatic genomic changes. The first occurs during the transition from free-living bacterium to a facultative symbiont and the second one with transition to an obligate symbiont. During these processes, bacterial genomes first undergo a dramatic decrease of coding density due to large-scale inactivation of the genes. This step is followed by removal of the inactivated genes and restoration of the coding density. Our set of new Wolbachia strains fit well into this scenario. As shown in Figure 5 most of the supergroup F genomes display relatively high coding density between $78 \%$ and $84 \%$. These genomes carry transposases and mobile elements, and with exception of $w C l e$, also a relatively rich repertoire of recombination/repair genes (Supplementary data 4). In contrast, the wMmer genome shows a considerable drop in coding density to $65 \%$. While its size is comparable to the former strains, it contains a significantly lower gene number, indicating large-scale deactivation. Finally, the wMeur1 genome restores the coding density to $76 \%$, apparently due to removal of its deactivated regions. Its position in this evolutionary spectrum, loss of most of the recombination/repair systems, and presumed metabolic role in pantothenate provision provide strong evidence that wMeur1 is the first known insect-associated Wolbachia strain which completed the transition to obligate mutualist.

\section{Acknowledgments}

This work was supported by the Grant Agency of the Czech Republic (grant 20-07674S to V.H.). We would like to acknowledge the sequencing services of the DNA Services of Roy J. Carver Biotechnology Center, University of Illinois at Urbana-Champaign, Illinois, USA. Access to computing and storage facilities owned by parties and projects contributing to the National Grid Infrastructure MetaCentrum provided under the programme "Projects of Large Research, Development, and Innovations Infrastructures" (CESNET LM2015042), is greatly appreciated. We thank Joel J. Brown for his language corrections, and Daniel R. Gustafsson (Guangdong Academy of Sciences) for providing us samples of M. eurysternus from China, France, Japan and Sweden for amplicon analysis.

\section{References}

1. Scholz M, Albanese D, Tuohy K, Donati C, Segata N, Rota-Stabelli O. Large scale genome reconstructions illuminate Wolbachia evolution. Nature Communications. 2020;11(1).

2. Lo N, Casiraghi M, Salati E, Bazzocchi C, Bandi C. How many Wolbachia supergroups exist? Molecular Biology and Evolution. 2002;19(3):341-6. 
3. Ferri E, Bain O, Barbuto M, Martin C, Lo N, Uni S, et al. New Insights into the Evolution of Wolbachia Infections in Filarial Nematodes Inferred from a Large Range of Screened Species. Plos One. 2011;6(6).

4. Lefoulon E, Clark T, Borveto F, Perriat-Sanguinet M, Moulia C, Slatko B, et al. Pseudoscorpion Wolbachia symbionts: diversity and evidence for a new supergroup S. Bmc Microbiology. 2020;20(1).

5. Baldo L, Hotopp J, Jolley K, Bordenstein S, Biber S, Choudhury R, et al. Multilocus sequence typing system for the endosymbiont Wolbachia pipientis. Applied and Environmental Microbiology. 2006;72(11):7098-110.

6. Hosokawa T, Koga R, Kikuchi $Y$, Meng X, Fukatsu T. Wolbachia as a bacteriocyte-associated nutritional mutualist. Proceedings of the National Academy of Sciences of the United States of America. 2010;107(2):769-74.

7. Ishmael N, Hotopp J, loannidis P, Biber S, Sakamoto J, Siozios S, et al. Extensive genomic diversity of closely related Wolbachia strains. Microbiology-Sgm. 2009;155:2211-22.

8. Driscoll T, Verhoeve V, Brockway C, Shrewsberry D, Plumer M, Sevdalis S, et al. Evolution of Wolbachia mutualism and reproductive parasitism: insight from two novel strains that co-infect cat fleas. Peerj. 2020;8.

9. Gerth M, Gansauge M, Weigert A, Bleidorn C. Phylogenomic analyses uncover origin and spread of the Wolbachia pandemic. Nature Communications. 2014;5.

10. Lefoulon E, Clark T, Guerrero R, Canizales I, Cardenas-Callirgos J, Junker K, et al. Diminutive, degraded but dissimilar: Wolbachia genomes from filarial nematodes do not conform to a single paradigm. Microbial Genomics. 2020;6(12).

11. Panaram K, Marshall J. F supergroup Wolbachia in bush crickets: what do patterns of sequence variation reveal about this supergroup and horizontal transfer between nematodes and arthropods? Genetica. 2007;130(1):53-60.

12. Keiser P, Coulibaly Y, Kubofcik J, Diallo A, Klion A, Traore S, et al. Molecular identification of Wolbachia from the filarial nematode Mansonella perstans. Molecular and Biochemical Parasitology. 2008;160(2):123-8.

13. Zimmermann B, Cardoso G, Bouchon D, Pezzi P, Palaoro A, Araujo P. Supergroup F Wolbachia in terrestrial isopods: Horizontal transmission from termites? Evolutionary Ecology. 2021;35(2):165-82.

14. Kyei-Poku G, Colwell D, Coghlin P, Benkel B, Floate K. On the ubiquity and phylogeny of Wolbachia in lice. Molecular Ecology. 2005;14(1):285-94.

15. Allen JM, Burleigh JG, Light JE, Reed DL. Effects of $16 \mathrm{~S}$ rDNA sampling on estimates of the number of endosymbiont lineages in sucking lice. PeerJ. 2016;4:e2187.

16. Boyd BM, Allen JM, de Crécy-Lagard V, Reed DL. Genome Sequence of Candidatus Riesia pediculischaeffi, Endosymbiont of Chimpanzee Lice, and Genomic Comparison of Recently Acquired Endosymbionts from Human and Chimpanzee Lice. G3: Genes|Genomes|Genetics. 2014;4(11):2189-95.

17. Boyd B, Allen J, Koga R, Fukatsu T, Sweet A, Johnson K, et al. Two Bacterial Genera, Sodalis and Rickettsia, Associated with the Seal Louse Proechinophthirus fluctus (Phthiraptera: Anoplura). Applied and Environmental Microbiology. 2016;82(11):3185-97.

18. Fukatsu T, Hosokawa T, Koga R, Nikoh N, Kato T, Hayama S, et al. Intestinal endocellular symbiotic bacterium of the macaque louse Pedicinus obtusus: Distinct endosymbiont origins in anthropoid primate lice and the old world monkey louse. Appl Environ Microbiol. 2009;75(11):3796-9.

19. Hypsa V, Krizek J. Molecular evidence for polyphyletic origin of the primary symbionts of sucking lice (phthiraptera, anoplura). Microb Ecol. 2007;54(2):242-51.

20. Rihova J, Novakova E, Husnik F, Hypsa V. Legionella Becoming a Mutualist: Adaptive Processes Shaping the Genome of Symbiont in the Louse Polyplax serrata. Genome Biology and Evolution. 2017;9(11):2946-57. 
21. Rihova J, Batani G, Rodriguez-Ruano S, Martinu J, Vacha F, Novakova E, et al. A new symbiotic lineage related to Neisseria and Snodgrassella arises from the dynamic and diverse microbiomes in sucking lice. Molecular Ecology. 2021;30(9):2178-96.

22. Allen JM, Reed DL, Perotti MA, Braig HR. Evolutionary relationships of "Candidatus Riesia spp.," endosymbiotic enterobacteriaceae living within hematophagous primate lice. Appl Environ Microbiol. 2007;73(5):1659-64.

23. de Moya R, Yoshizawa K, Walden K, Sweet A, Dietrich C, Kevin P. Phylogenomics of Parasitic and Nonparasitic Lice (Insecta: Psocodea): Combining Sequence Data and Exploring Compositional Bias Solutions in Next Generation Data Sets. Systematic Biology. 2021;70(4):719-38.

24. Smith VS. The Chewing Lice: World Checklist and Biological Overview.-R. D. Price, R. A. Hellenthal, R. L. Palma, K. P. Johnson, D. H. Clayton. 2003. Illinois Natural History Survey Special Publication 24. $x+501$ pp. ISBN 1-882932-08-0.

25. Marcondes CB, Linardi PM. Sucking and Chewing Lice. In: Marcondes CB, editor. Arthropod Borne Diseases. Cham: Springer International Publishing; 2017. p. 503-15.

26. Alickovic L, Johnson K, Boyd B. The reduced genome of a heritable symbiont from an ectoparasitic feather feeding louse. Bmc Ecology and Evolution. 2021;21(1).

27. Martinu J, Sychra O, Literak I, Capek M, Gustafsson D, Stefka J. Host generalists and specialists emerging side by side: an analysis of evolutionary patterns in the cosmopolitan chewing louse genus Menacanthus. International Journal For Parasitology. 2015;45(1):63-73.

28. Leinonen R, Sugawara H, Shumway M, C INSD, C INSD. The Sequence Read Archive. Nucleic Acids Research. 2011;39:D19-D21.

29. Brown J, Rodriguez-Ruano S, Poosakkannu A, Batani G, Schmidt J, Roachell W, et al. Ontogeny, species identity, and environment dominate microbiome dynamics in wild populations of kissing bugs (Triatominae). Microbiome. 2020;8(1).

30. Parada A, Needham D, Fuhrman J. Every base matters: assessing small subunit rRNA primers for marine microbiomes with mock communities, time series and global field samples. Environmental Microbiology. 2016;18(5):1403-14.

31. Quince C, Lanzen A, Davenport R, Turnbaugh P. Removing Noise From Pyrosequenced Amplicons. Bmc Bioinformatics. 2011;12.

32. Edgar RC. UPARSE: highly accurate OTU sequences from microbial amplicon reads. Nature Methods. 2013;10(10):996-+.

33. Caporaso JG, Kuczynski J, Stombaugh J, Bittinger K, Bushman FD, Costello EK, et al. QIIME allows analysis of high-throughput community sequencing data. Nature Methods. 2010;7(5):335-6.

34. Team R. RStudio: Integrated Development for R.: RStudio, PBC, Boston, MA URL http://www.rstudio.com/ . 2020.

35. McMurdie PJ, Holmes S. phyloseq: An R Package for Reproducible Interactive Analysis and Graphics of Microbiome Census Data. Plos One. 2013;8(4).

36. B. B. BBMap short-read aligner, and other bioinformatics tools.: University of California, Berkeley, CA . 2015.

37. Camacho C, Coulouris G, Avagyan V, Ma N, Papadopoulos J, Bealer K, et al. BLAST plus : architecture and applications. Bmc Bioinformatics. 2009;10.

38. Allen J, LaFrance R, Folk R, Johnson K, Guralnick R. aTRAM 2.0: An Improved, Flexible Locus Assembler for NGS Data. Evolutionary Bioinformatics. 2018;14.

39. Aziz RK, Bartels D, Best AA, DeJongh M, Disz T, Edwards RA, et al. The RAST Server: rapid annotations using subsystems technology. BMC Genomics. 2008;9:75.

40. Arndt D, Grant J, Marcu A, Sajed T, Pon A, Liang Y, et al. PHASTER: a better, faster version of the PHAST phage search tool. Nucleic Acids Research. 2016;44(W1):W16-W21. 
41. Syberg-Olsen $M$, Keeling $P$, McCutcheon J, Husnik F. Pseudofinder. GitHub repository: https://github.com/filip-husnik/pseudofinder/ . 2020.

42. Seemann T. Prokka: rapid prokaryotic genome annotation. Bioinformatics. 2014;30(14):2068-9.

43. Buchfink B, Xie C, Huson D. Fast and sensitive protein alignment using DIAMOND. Nature Methods. 2015;12(1):59-60.

44. Huerta-Cepas J, Forslund K, Coelho L, Szklarczyk D, Jensen L, von Mering C, et al. Fast GenomeWide Functional Annotation through Orthology Assignment by eggNOG-Mapper. Molecular Biology and Evolution. 2017;34(8):2115-22.

45. Conway J, Lex A, Gehlenborg N. UpSetR: an R package for the visualization of intersecting sets and their properties. Bioinformatics. 2017;33(18):2938-40.

46. Jolley K, Maiden M. BIGSdb: Scalable analysis of bacterial genome variation at the population level. Bmc Bioinformatics. 2010;11.

47. Edgar R. MUSCLE: a multiple sequence alignment method with reduced time and space complexity. Bmc Bioinformatics. 2004;5:1-19.

48. Trifinopoulos J, Nguyen L, von Haeseler A, Minh B. W-IQ-TREE: a fast online phylogenetic tool for maximum likelihood analysis. Nucleic Acids Research. 2016;44(W1):W232-W5.

49. Emms D, Kelly S. OrthoFinder: phylogenetic orthology inference for comparative genomics. Genome Biology. 2019;20(1).

50. Castresana J. Selection of conserved blocks from multiple alignments for their use in phylogenetic analysis. Molecular Biology and Evolution. 2000;17(4):540-52.

51. Lartillot N, Rodrigue N, Stubbs D, Richer J. PhyloBayes MPI: Phylogenetic Reconstruction with Infinite Mixtures of Profiles in a Parallel Environment. Systematic Biology. 2013;62(4):611-5.

52. Lartillot N, Brinkmann H, Philippe H. Suppression of long-branch attraction artefacts in the animal phylogeny using a site-heterogeneous model. Bmc Evolutionary Biology. 2007;7.

53. Yoon S, Ha S, Lim J, Kwon S, Chun J. A large-scale evaluation of algorithms to calculate average nucleotide identity. Antonie Van Leeuwenhoek International Journal of General and Molecular Microbiology. 2017;110(10):1281-6.

54. Darling A, Mau B, Perna N. progressiveMauve: Multiple Genome Alignment with Gene Gain, Loss and Rearrangement. Plos One. 2010;5(6).

55. Kearse M, Moir R, Wilson A, Stones-Havas S, Cheung M, Sturrock S, et al. Geneious Basic: An integrated and extendable desktop software platform for the organization and analysis of sequence data. Bioinformatics. 2012;28(12):1647-9.

56. Kanehisa M, Sato $Y$, Kawashima M, Furumichi M, Tanabe M. KEGG as a reference resource for gene and protein annotation. Nucleic Acids Research. 2016;44(D1):D457-D62.

57. Shigenobu S, Watanabe H, Hattori M, Sakaki Y, Ishikawa H. Genome sequence of the endocellular bacterial symbiont of aphids Buchnera sp APS. Nature. 2000;407(6800):81-6.

58. Wernegreen JJ. Genome evolution in bacterial endosymbionts of insects. Nat Rev Genet. 2002;3(11):850-61.

59. Nikoh N, Hosokawa T, Moriyama M, Oshima K, Hattori M, Fukatsu T. Evolutionary origin of insectWolbachia nutritional mutualism. Proceedings of the National Academy of Sciences of the United States of America. 2014;111(28):10257-62.

60. Darby AC, Armstrong SD, Bah GS, Kaur G, Hughes MA, Kay SM, et al. Analysis of gene expression from the Wolbachia genome of a filarial nematode supports both metabolic and defensive roles within the symbiosis. Genome Research. 2012;22(12):2467-77.

61. Smith W, Oakeson K, Johnson K, Reed D, Carter T, Smith K, et al. Phylogenetic analysis of symbionts in feather-feeding lice of the genus Columbicola: evidence for repeated symbiont replacements. Bmc Evolutionary Biology. 2013;13. 
62. Fisher R, Henry L, Cornwallis C, Kiers E, West S. The evolution of host-symbiont dependence. Nature Communications. 2017;8.

63. Gerth M, Bleidorn C. Comparative genomics provides a timeframe for Wolbachia evolution and exposes a recent biotin synthesis operon transfer. Nature Microbiology. 2017;2(3).

64. Newton I, Rice D. The Jekyll and Hyde Symbiont: Could Wolbachia Be a Nutritional Mutualist? Journal of Bacteriology. 2020;202(4).

65. Kirkness EF, Haas BJ, Sun WL, Braig HR, Perotti MA, Clark JM, et al. Genome sequences of the human body louse and its primary endosymbiont provide insights into the permanent parasitic lifestyle. Proceedings of the National Academy of Sciences of the United States of America. 2010;107(27):1216873.

66. Lo $\mathrm{W}$, Huang $\mathrm{Y}$, Kuo $\mathrm{C}$. Winding paths to simplicity: genome evolution in facultative insect symbionts. Fems Microbiology Reviews. 2016;40(6):855-74. 